\title{
Estimation of Salivary Immunoglobulin A and Total Salivary Protein in Patients with Recurrent Aphthous Ulcer
}

\author{
Reshma Ramachandran ${ }^{1 *}$ and Anuradha Pai $^{2}$ \\ ${ }^{1}$ Department of Oral Medicine and Radiology, The Oxford Dental College, India \\ ${ }^{2}$ H.O.D and Professor, Department of Oral Medicine and Radiology, The Oxford Dental \\ College, India \\ *Corresponding Author: Reshma Ramachandran, Department of Oral Medicine and \\ Radiology, The Oxford Dental College, India.
}

Received: February 06, 2021

Published: February 27, 2021

(C) All rights are reserved by Reshma

Ramachandran and Anuradha Pai.

\begin{abstract}
Background: Recurrent aphthous ulceration is amongst the not uncommon ulcerative, inflammatory, oral mucosal conditions whose precise etiopathogenesis remain obscure. Several local and systemic factors have been postulated as the possible predisposing agents for RAS. The presence of immune factors at the site locally, have been seen to play a pivotal role in fortification in counteraction to oral diseases.

Aims and Objectives

- To estimate the salivary immunoglobulin A levels in minor recurrent aphthous ulcer patients and in healthy volunteers.

- To estimate the total salivary protein levels in both study and control group.

- To compare the levels of IgA and total salivary proteins in minor recurrent aphthous ulcer patients and in healthy volunteers.

Materials and Method: 60 participants were enrolled into the study and were allocated amongst two groups. Group I were included with 30 patients diagnosed with minor recurrent aphthous ulcers and group II with 30 healthy controls. Whole saliva was collected and centrifuged to determine salivary immunoglobulin A and total salivary proteins levels in both the groups.

Results: In case group they showed a significantly higher levels of salivary IgA and total proteins compared with the healthy control group ( $\mathrm{p}<0.001$ ) and hence proving that the salivary immunoglobulin A may help in the diagnosing of minor RAS.

Conclusion: A strong correlation were found between salivary IgA which may help in the pathogenesis of minor recurrent aphthous ulcers and this may be used as a parameter to assess the mucosal immune status.

Keywords: RAS; Salivary Immunoglobulin A; Total Salivary Proteins
\end{abstract}

\section{Introduction}

Recurrent aphthous stomatitis (RAS) is considered to be amongst common ulcerative lesions of the oral cavity. It is characterized by recurring painful ulcers of the mouth that are round or ovoid covered by grayish white fibrin pseudo-membrane and surrounded by inflammatory halos [1,2]. The term aphthous is derived from a Greek word 'aphtha' meaning ulceration [2,3]. It is amongst the diseases with and unknown mechanism of action and is among the most challenging problems faced by the affected persons and clinicians alike [4].
The precise etiology of RAS is a myth. The disease incidence has a range of $5 \%$ to $50 \%$ amongst general populations [1,5]. The potential trigger factors considered are local factors such as trauma in individual who are genetically susceptible to RAS, microbial factors, nutritional factors, such as deficiency of folate and B- complex vitamins, immunologic factors, psychological stress, and allergy to dietary constituents. Extensive research has focused predominantly on immunologic factors, but a definitive etiology is not known [6].

Local immune factors play a role in protection against oral disease which may be related to immunoglobulin A (IgA). IgA belongs 
to the majority immunoglobulin group which is found in mucous secretion. Antibodies present in mucous secretion exclusively IgA, are devoted to micro-organism and diminish their freedom of movement and adhesive characteristics and are well-thought-out as the primary defense of the oral cavity. Salivary IgA is an excellent indicator of oral mucosa immune status [7].

Total protein is a dynamic part of saliva and is liable for most of its roles like lubrication, physical safety, cleansing, buffering, conservation of tooth integrity and antiseptic action. Their level in the oral cavity is subjected to constant fluctuation which depend on numerous factors [8].

Secretory immunoglobulin A (IgA-s) is an essential factor to gauge the auto immune status of the mucosa, with the advantage of being measurable by non-invasive methods and without patient discomfort. It is the major class of immunoglobulin seen in mucous secretions, and is accountable for a blockade against a number of infectious, environmental allergens and carcinogenic substances and it also aids in many inborn protection mechanism.

$\operatorname{IgA}$ is the subsequent most profuse immunoglobulin in the human serum at the second position and prevails in the saliva in its dimeric form which better resist proteolysis in environments such as the mouth. By considering that the salivary immune system is dynamically tangled in the pathogenesis of recurrent aphthous ulcers, measuring IgA-s changes might have a valuable role in prediction of the onset and management of the disease [9].

So far there is sparse literature regarding the relationship between total protein and recurrent aphthous ulcer. Hence the present study is designed to estimate the salivary immunoglobulin A levels and the total salivary proteins in recurrent aphthous ulcers and in healthy control group.

\section{Materials and Methods}

The study consists of 60 patients visiting the department of Oral medicine and Radiology in the age group of 18-50 years with single or multiple minor RAS of less than 48 hours duration. The ethical clearance was obtained from institutional ethical board.

A total of 60 participants were enrolled in the study and divided into two groups, group I is the study group which consist of 30 RAS patients and group II include 30 healthy controls.

\section{Exclusion criteria}

- Patients with history of previous malignancy.

- $\quad$ Ulcers as manifestation of systemic disease.

- Chronic systemic disease

- Drug therapy in the week prior to study

- Patients who have received dental treatment.

An informed consent was taken before the saliva collection procedure and unstimulated whole saliva measuring $1 \mathrm{ml}$ was collected by spit method in the calibrated measuring cup. The samples were centrifuged at $3000 \mathrm{rpm}$ for 15 minutes and supernatants are collected and stored at $-20^{\circ} \mathrm{C}$ before transferring for lab analysis. Care was taken to see that patient's does not consume food, smoke or chew gum at least one hour before the saliva collection procedure. The samples were then analyzed for salivary immunoglobulin A by immune Turbidimetric method and total salivary proteins by Biuret method.

Salivary IgA and total salivary protein levels in both the groups were tabulated and data was statistically analyzed by using Independent student $t$ test and Mann Whitney test.

\section{Results}

In the study of 60 individuals, the mean age of study group was $27 \pm 3.76$ years where as in control group it was $27.70 \pm 3.90$ years with a range of 21-36 years in study group and 19-43 years in the control group with a $\mathrm{p}$ value of 0.73 . Out of 30 participants 12 (40\%) were males and 18 (60\%) were females in the study group where as in control group 16 (53.3\%) were males and 14 (46.7\%) were females with a p value of 0.30 (Table 1 ).

\begin{tabular}{|c|c|c|c|c|c|c|}
\hline \multicolumn{7}{|c|}{ Age and Gender Distribution among study and Control } \\
groups \\
\hline \multirow{2}{*}{ Variables } & \multirow{2}{*}{ Category } & \multicolumn{2}{|c|}{ Control } & \multicolumn{2}{c|}{ Case } & \multirow{2}{*}{ P-Value } \\
\cline { 3 - 7 } & Mean & SD & Mean & SD & \\
\hline \multirow{2}{*}{ Age } & Mean and SD & 27.70 & 3.90 & 27.37 & 3.76 & \multirow{2}{*}{$0.73^{\mathrm{a}}$} \\
\cline { 2 - 7 } & Range & \multicolumn{2}{|c|}{$19-43$} & $21-36$ & \\
\hline \multirow{2}{*}{ Gender } & & $\mathrm{n}$ & $\%$ & $\mathrm{n}$ & $\%$ & \\
\hline & Males & 16 & $53.3 \%$ & 12 & $40.0 \%$ & $0.30^{\mathrm{b}}$ \\
\hline & Females & 14 & $46.7 \%$ & 18 & $60.0 \%$ & \\
\hline
\end{tabular}

Table 1: Age and gender distribution of study participants.

Note: a. Mann Whitney U Test

b. Chi Square Test. 
The mean salivary IgA level in study group is $45.63 \pm 2.14$ where as in control group it is $26.30 \pm 1.47$ with a p value $<0.001$ and it is statistically significant (Table 2) (Graph 1 ). The mean salivary total proteins level in study group is $0.047 \pm 0.068$ where as in control group is $0.019 \pm 0.009$ with a $\mathrm{p}$ value $<0.001$ and it is statistically significant (Table 3) (Graph 2).
Out of 30 controls 7 participants were in the age group of 20-25 years and with a mean $26.71 \pm 1.71 .19$ participants were in the age group 26-30 years with a mean $26.00 \pm 1.60$ and 4 subjects were more than 30 years with a mean of $27.00 \pm 1.16$ with a p value of 0.33 .

\begin{tabular}{|c|c|c|c|c|c|c|c|}
\hline \multicolumn{7}{|c|}{ Comparison of mean Salivary IgA (mg/d) between study and Control using Independent Student t Test } \\
\hline Parameters & Group & N & Mean & SD & Mean Diff & t & P-Value \\
\hline Salivary IgA & Control & 30 & 26.30 & 1.47 & -19.33 & -40.809 & $<0.001^{*}$ \\
\cline { 2 - 8 } & Case & 30 & 45.63 & 2.14 & & & \\
\hline
\end{tabular}

Table 2: Comparison of mean salivary IgA (mg/dl) between study and control.

$$
\text { * - Statistically Significant. }
$$

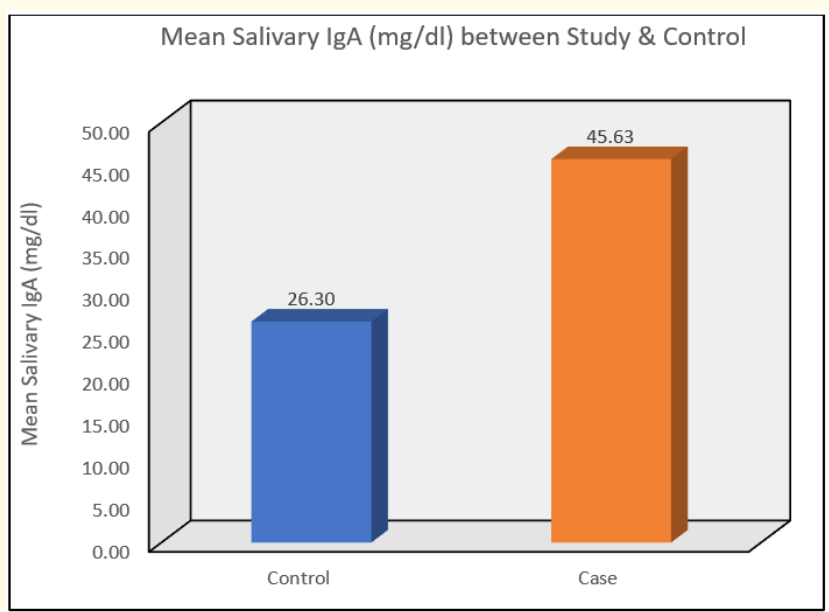

Graph 1: Comparison of mean salivary IgA (mg/dl) between study and control.

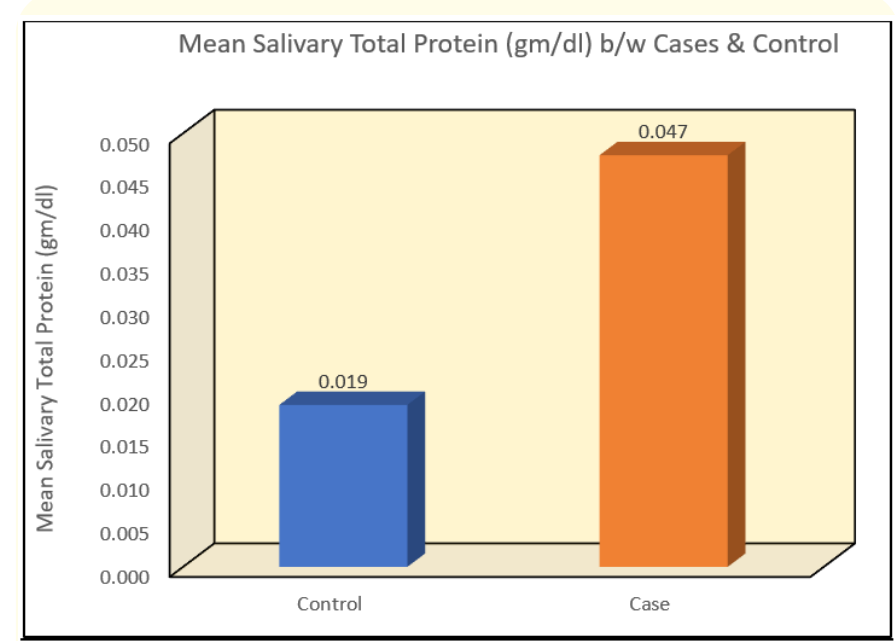

Graph 2: Comparison of mean salivary total protein levels between study and control group.

\begin{tabular}{|c|c|c|c|c|c|c|c|}
\hline \multicolumn{6}{|c|}{ Comparison of mean Salivary Total Protein levels (g/dl) between study and Control using Mann Whitney Test } \\
\hline Parameters & Group & N & Mean & SD & Mean Diff & Z & P-Value \\
\hline \multirow{2}{*}{ Sal. Total Protein } & Control & 30 & 0.019 & 0.009 & -0.029 & -5.392 & $<0.001^{*}$ \\
\cline { 2 - 8 } & Case & 30 & 0.047 & 0.068 & & & \\
\end{tabular}

Table 3: Comparison of mean salivary total protein levels between study and control.

$$
\text { * - Statistically Significant. }
$$

Among 30 patients in the study group, 9 patients were in the age group of 20-25 years with a mean of $46.56 \pm 1.9$ and 16 were in the age group of 26-30 years with a mean of $45.88 \pm 2.06$ and 5 subjects were more than 30 years of age with a mean of $43.20 \pm 2.39$ with a p value of 0.01 and it is statistically significant (Table 4) (Graph 3).
7 participants out of 30 were in the age group 20-25 years with a mean of $0.017 \pm 0.005$ and 19 subjects were in age group 2630 years with a mean $0.020 \pm 0.011$ and 4 participants were more than 30 years with a mean of $0.015 \pm 0.006$ with a p value of 0.54 . 


\begin{tabular}{|c|c|c|c|c|c|c|c|c|}
\hline \multicolumn{9}{|c|}{$\begin{array}{c}\text { Age wise comparison of mean Salivary IgA (in mg/dl) levels among study group using one-way ANOVA test followed by } \\
\text { Tukey's Post hoc Analysis }\end{array}$} \\
\hline Age Grp & $\mathbf{N}$ & Mean & SD & Min & Max & P-Value ${ }^{a}$ & Sig. Diff & P-Value ${ }^{b}$ \\
\hline $20-25$ yrs & 9 & 46.56 & 1.01 & 45 & 48 & \multirow[t]{3}{*}{$0.01^{*}$} & A1 vs A2 & 0.66 \\
\hline $26-30$ yrs & 16 & 45.88 & 2.06 & 42 & 50 & & A1 vs A3 & $0.009^{*}$ \\
\hline$>30$ yrs & 5 & 43.20 & 2.39 & 40 & 46 & & $\mathrm{~A} 2$ vs $\mathrm{A} 3$ & $0.03^{*}$ \\
\hline
\end{tabular}

Table 4: Age wise comparison of mean salivary IgA levels among study group.

* - Statistically Significant

Note: A1 - 20 - 25 yrs, A2 - 26-30 yrs, A3 - > 30 yrs

a. P-value derived by one-way ANOVA test, b. P-value dervied by Tukey's post hoc Test.

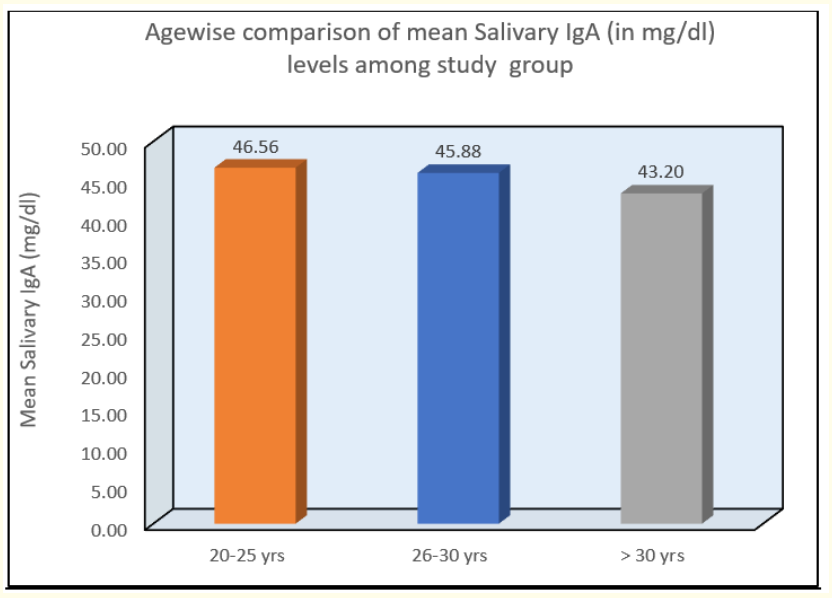

Graph 3: Age wise comparison of mean salivary IgA levels among study group.
9 participants out of 30 were in the age group of 20-25 years with a mean of $0.059 \pm 0.083,16$ participants were in the age $26-30$ years with a mean of $0.049 \pm 0.070$ and 5 participants were more than 30 years with a mean of $0.021 \pm 0.001$ with a p value of 0.001 and they are statistically significant (Table 5) (Graph 4). Out of 30 controls 16 were males with a mean of $26.31 \pm 1.62$ and 14 were females with a mean of $26.29 \pm 1.33$ with a p value of 0.96 .

12 patients out of 30 were males with a mean of $44.00 \pm 2.05$ and 18 were females with a mean of $46.72 \pm 1.41$ and $p$ value less than 0.001 (statistically significant) (Table 6). 16 participants were males out of 30 with a mean of $0.019 \pm 0.013$ and 14 were females with a mean of $0.019 \pm 0.004$ with a p value of 0.98 . 12 were males out of 30 patients with a mean of $0.052 \pm 0.082$ and 18 were female with a mean of $0.045 \pm 0.059$ with $p$ value of 0.61 .

\begin{tabular}{|c|c|c|c|c|c|c|c|c|}
\hline \multicolumn{9}{|c|}{$\begin{array}{c}\text { Age wise comparison of mean Salivary Total Protein (in gm/dl) among study group using Kruskal Wallis test followed } \\
\text { by Mann Whitney Post hoc Analysis }\end{array}$} \\
\hline Age Grp & $\mathbf{N}$ & Mean & SD & Min & Max & P-Value & Sig. Diff & P-Value $^{\mathrm{b}}$ \\
\hline $20-25$ yrs & 9 & 0.059 & 0.083 & 0.02 & 0.28 & \multirow[t]{3}{*}{$0.001^{*}$} & A1 vs A2 & 0.75 \\
\hline $26-30$ yrs & 16 & 0.049 & 0.070 & 0.02 & 0.31 & & A1 vs A3 & $0.007^{*}$ \\
\hline$>30$ yrs & 5 & 0.021 & 0.001 & 0.02 & 0.02 & & A2 vs A3 & 0.003* \\
\hline
\end{tabular}

Table 5: Age wise comparison of mean salivary total proteins levels among study group.

* - Statistically Significant

Note: A1 - 20 - 25 yrs, A2 - 26-30 yrs, A3 - > 30 yrs.

a. P-value derived by Kruskal Wallis test, b. P-value dervied by Mann Whitney Post hoc Test. 


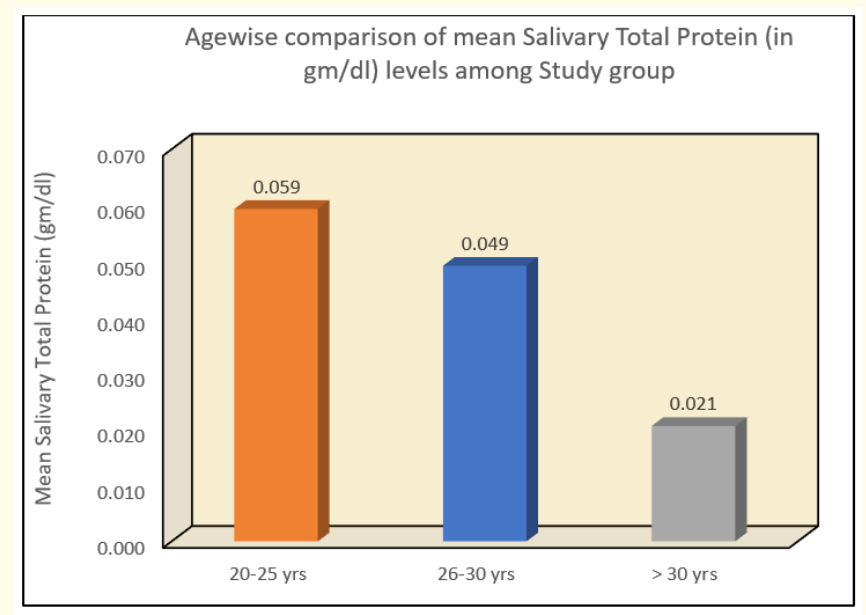

Graph 4: Age wise comparison of mean salivary total proteins levels among study group.
The correlation co-efficient of salivary IgA in control and study group is 1 where as in total salivary protein in control the co- efficient is -0.05 and in study group it is 0.22 which implies that salivary immunoglobulin A have a very strong correlation with minor recurrent aphthous ulcer whereas total salivary proteins have a weak correlation with the disease (Table 7).

\section{Discussion}

Recurrent aphthous ulceration also known as canker sores represents the second most common type of oral ulcerations after traumatic ulceration. The classic presentation of RAS is recurrent, self-limiting ulcers which primarily distress the non-keratinized oral mucosa [10]. They are categorized into the clinical variation namely minor, major and herpetiform. Minor aphthous accounts in around $70-90 \%$ of RAS patients presenting the most common form of aphthous ulcerations [11].

\begin{tabular}{|c|c|c|c|c|c|c|}
\hline \multicolumn{6}{|c|}{ Gender wise comparison of mean Salivary IgA (in mg/dl) levels among study group using Independent Student $\mathbf{t}$ Test } \\
\hline Gender & N & Mean & SD & Mean Diff & t & P-Value \\
\hline Males & 12 & 44.00 & 2.05 & -2.72 & -4.332 & $<0.001^{*}$ \\
\cline { 1 - 4 } Females & 18 & 46.72 & 1.41 & & & \\
\hline
\end{tabular}

Table 6: Gender wise comparison of mean salivary IgA in study group.

* - Statistically Significant

\begin{tabular}{|c|c|c|c|c|}
\hline \multicolumn{5}{|c|}{ Spearman's correlation coefficients b/w 2 variables among control and study group } \\
\hline Group & Variables & Values & Sal. IgA & Sal. TP \\
\hline Control & Sal. IgA & Rho & 1 & -0.05 \\
\cline { 3 - 5 } & & P-Value &.. & 0.81 \\
\hline Case & Sal. IgA & Rho & 1 & 0.22 \\
\cline { 3 - 5 } & & P-Value &.. & 0.25 \\
\hline
\end{tabular}

The correlation coefficients are denoted by 'Rho'

Minus sign denotes negative correlation

Correlation coefficient range

$$
0.0 \text { - No Correlation }
$$

0.01 - 0.20 - Very Weak Correlation

0.21 - 0.40 - Weak Correlation

0.41 - 0.60 - Moderate Correlation

0.61 - 0.80 - Strong Correlation

0.81 - 1.00 - Very Strong Correlation.

Table 7: Spearman's correlation co-efficient between two variables among control and study group. 
Secretory immunoglobulin A (IgA-s) has the advantage of being measurable by non-invasive methods and without patient discomfort. It is responsible as a barrier against a number of infectious, environmental allergies and carcinogenic substances and it also helps in several in born protection mechanism [7].

Considering the fact that immunity has some regulatory effects on the development of recurrent ulcers, salivary IgA provides defense, considering its role in other body parts like in gastro intestinal tract, intestines and respiratory tract [12].

IgA embodies the second most profuse immunoglobulin in the human serum and overcomes in the saliva in its dimeric form which superior resist proteolysis in environment such as mouth.

Antibodies prevailing in mucosal secretions, especially IgA are attached to microorganism and reduces their mobility and adhesive properties and are reflected as the first line of defense in oral cavity [13].

Accepting the fact that salivary immune system is actively involved in the pathogenesis of recurrent aphthous ulcers, measuring salivary IgA and total protein changes may have a valuable role in prediction of the onset and management of disease.

The present study aimed to estimate the salivary immunoglobulin A and total salivary proteins in minor RAS and in healthy controls. 30 patients with known history of minor RAS were diagnosed based on the major criteria with the absence of any other systemic disease or conditions and presenting with single and multiple ulcers were included in the study. Minor aphthous were selected as they are more common than the major and herpetiform ulcers.

Unstimulated saliva was collected by spit method in a calibrated measuring cup from 60 participants (30 RAS and 30 healthy controls) to assess the salivary IgA levels and total salivary proteins. A detailed case history and informed constant was taken from all the participants prior to our study.

In our study, female predominance was noted in the study group which account for $60 \%$ (18). This is in accordance with Martinez., et al. and Rajmane., et al. study where majority of the patients were females $[10,14]$. Females are more prone to stress and emotional situation which can affect their immune response. They seek medical examination more frequently than males. The hormonal changes during pregnancy and menstruation also play an important role. Maximum number of patient belongs to 21-30 years of age group which is correlated with the study conducted by Kareem., et al. in which about $80 \%$ patients developed the condition before 30 years of age. The highest incidence is among young people however the severity and frequency of ulcers decrease with age [15].

A high prevalence and severity of the disease has been found in student with a high socioeconomic background. This is because of psychological stress which acts as a triggering factor for RAS and is typically observed during stressful situations such as academic load in professional students and also in any other significant changes in life. This in accordance with Abdullah., et al. study, where majority of the patients were students [16].

In our study there was significant relationship between the age and gender of the patients with the levels of salivary IgA. But it is not so in case of total salivary proteins. In Mortinez., et al. (2007) study where they assessed secretory IgA level total proteins and the salivary flow in 20 aphthous ulcer patients where they found no significant relationship between age and gender with salivary IgA and total proteins.

The present study, the level of salivary IgA in study group was significantly higher than the control group which is in accordance with Pakfetrat., et al, Martinez., et al, Mohammed., et al. This could reflect an important role for salivary IgA in pathogenesis of RAS. Four mechanisms could be proposed:

- Firstly, the presence of a threshold level of salivary IgA above which direct destruction of keratinocytes may commence.

- Secondly indirect destruction through IgA immune complex- mediated mechanism, although this type of uncontrolled immune response occurs in the circulation;

- Thirdly, these IgA antibodies are auto antibodies against oral mucosal cell antigens, or they may be produced against a foreign antigen that is immunologically cross-reactive with a component of oral self-tissues.

- Fourthly, these elevated levels might be a normal local immune response (innate or adaptive) to neutralize the etiological factors that have already caused epithelial destruction.

The principle mechanism of protective immunity against antigens in mucosal lumen is antibody- mediated neutralization which is dominated by IgA. 
In our study the level of salivary total protein for study group was significantly lower than the control group and it was contradictory with the results by Martinez., et al. where the total protein concentration did not vary when the patients were compared with and without lesions.

The results of this study suggest that there is a strong correlation between IgA-s and the pathogenesis of recurrent aphthous ulcer and a weak correlation of salivary total proteins with the lesion. IgA-s is implicated in recurrent aphthous ulcer and may play an important role in their defense mechanisms and it also helps in the pathogenesis of RAS. But the exact protective role of this immunoglobulin is still not clearly defined.

\section{Future Recommendations}

In future, long term studies with longer samples are required to determine the role of salivary immunoglobulin $\mathrm{A}$ and total proteins in minor recurrent aphthous ulcers, so that by measuring IgA-s and total protein changes may have a valuable role in prediction of the onset of the diseases and also help in better management of it.

\section{Conclusion}

RAS is one of the most common painful oral mucosal inflammatory ulcerative conditions and can cause difficulty on eating, swallowing and speaking and is known to hinder the quality of life and well-being to a certain extent. Due to numerous proposed etiologic factors and variety of treatment modalities, it has gathered a considerable amount of clinical and research attention.

However studies on longer series of patients for a longer duration may be required to determine the exact pathogenesis of minor RAS and the role of salivary IgA and total salivary protein in the disease mechanism.

\section{Bibliography}

1. Preeti L., et al. "Recurrent aphthous stomatitis". Journal of Oral and Maxillofacial Pathology: JOMFP 15.3 (2011): 252.

2. Swain N., et al. "Etiological factors of recurrent aphthous stomatitis: A common perplexity". Journal of Contemporary Dentistry 2.3 (2012): 96-100.

3. Ślebioda Z., et al. "Etiopathogenesis of recurrent aphthous stomatitis and the role of immunologic aspects: literature review". Archivum Immunologiae Et Therapiae Experimentalis 62.3 (2014): 205-215.
4. Porter SR., et al. "Recurrent aphthous stomatitis". Critical Reviews in Oral Biology and Medicine 9.3 (1998): 306-321.

5. Rivera C. "Essentials of recurrent aphthous stomatitis". Biomedical Reports 11.2 (2019): 47-50.

6. Queiroz SI., et al. "Recurrent aphthous ulceration: an epidemiological study of etiological factors, treatment and differential diagnosis". Anais Brasileiros De Dermatologia 93.3 (2018): 341-346.

7. Boras VV and Savage NW. "Recurrent aphthous ulcerative disease: presentations and management". Australian Dental Journal 52.1 (2007): 10-15.

8. Vivek V and Nair BJ. "Recurrent aphthous stomatitis: Current concepts in diagnosis and management". Journal of Indian Academy of Oral Medicine and Radiology 23.3 (2011): 232.

9. Gambhir RS., et al. "The prevalence of oral mucosal lesions in the patients visiting a dental school in Northern India in relation to sex, site and distribution: A retrospective study" (2011).

10. Sabbagh AH and Felemban MF. "Therapeutic Management of Recurrent Aphthous Stomatitis: A Review of the Growing Knowledge". Annals of International Medical and Dental Research 2.6 (2016): 1.

11. Brozović S., et al. "Serum IgA, IgG, IgM and salivary IgA in recurrent aphthous ulceration". Collegium Antropologicum 25.2 (2001): 633-637.

12. Güven 0. "Serum immunoglobulins in recurrent aphthous stomatitis". The Journal of Nihon University School of Dentistry 30.4 (1988): 297-301.

13. Mohammad R., et al. "Levels of salivary IgA inpatients with minor recurrent aphthous stomatitis: a matched case-control study". Clinical Oral Investigations 17.3 (2013): 975-980.

14. Martinez KdeO., et al. "Secretory A immunoglobulin, total proteins and salivary flow in Recurrent Aphthous Ulceration". Brazilian Journal of Otorhinolaryngology 73.3 (2007): 323-328.

15. A Kareem SAL and Ahmed KM. "Prevalence of Aphthous Ulceration in patients attending Oral Diagnosis Clinics at School of Dentistry/University of Sulaimani for four academic years (2010-2014)". IOSR Journal of Dental and Medical Sciences (IOSR-JDMS) 14 (2015): 80-84. 
16. Abdullah MJ. "Prevalence of recurrent aphthous ulceration experience in patients attending Piramird dental speciality in Sulaimani City". Journal of Clinical and Experimental Dentistry 5.2 (2013): e89.

\section{Assets from publication with us}

- Prompt Acknowledgement after receiving the article

- Thorough Double blinded peer review

- Rapid Publication

- Issue of Publication Certificate

- High visibility of your Published work

Website: www.actascientific.com/

Submit Article: www.actascientific.com/submission.php

Email us: editor@actascientific.com

Contact us: +919182824667 